\title{
Envejecimiento y género: Reconstruyendo los roles sociales de las personas mayores en los cuidados
}

Recibido: 30 de febrero 2019

Revisado: 30 de marzo 2019

Aprobado: 3 de mayo 2019

Marcelo Piña Morán

Chileno. Doctor en Estudios

Americanos, mención

Pensamiento y Cultura.

Labora en la Facultad de

Ciencias Sociales y

Económicas de la

Universidad Católica del

Maule, Talca, Chile. Sus

áreas de especialización son

Envejecimiento y Cultura; Trabajo Social y

Gerontología; Epistemología de la Investigación Social.

Correo electrónico: pina_moran@hotmail.com

Verónica Gómez Urrutia Chilena. Ph.D. in SociologyGender Studies. Labora en

la Facultad de Ciencias Sociales y Humanidades de la Universidad Autónoma de Chile, Talca, Chile. Sus áreas de especialización son el análisis de políticas públicas y legislación sobre género y familia en América Latina y la educación en ciudadanía. Correo electrónico: vgomezu@uautonoma.cl
Resumen: Este artículo aborda el tema de los roles sociales de las personas mayores desde la perspectiva de la gerontología social y, específicamente, la gerontología rupturista. La propuesta enfatiza la naturaleza diferenciada por género del envejecimiento, utilizando como eje de la discusión la temática de cuidados. Se argumenta que una (re)construcción inclusiva de los roles sociales en esta fase de la vida requiere plantear una ruptura con la mirada tradicional de las personas mayores como receptores pasivos de política dirigidas a ellos/as, en aras de considerar la necesidad de levantar información sobre la valoración subjetiva que los individuos realizan de la situación social en que los pone la vejez, las potencialidades de este periodo y los roles asociados a ella.

Palabras clave: género; envejecimiento; roles sociales; gerontología rupturista; trabajo de cuidados; teorías gerontológicas

\section{Aging and Gender: Reconstructing Older People's Social Roles in Care Work}

Abstract: This paper discusses the social roles of older people from a social gerontology perspective; particularly, critical gerontology. Considering the process of growing older as a gendered one, the example of care work is used to argue that an inclusive re-construction of older people's social roles must include a critical reflection on the intersection between gender and ideas on ageing and its potentialities. Such a reflection should include the need of gathering information on the subjective assessment individuals make on what it means to be old, the potentialities of this life stage and the roles associated to it, as opposed to consider older people the passive recipients of public policy aimed at this age group.

Key words: gender; ageing; social roles; rupturist gerontology; carework; gerontological theories 
1. La gerontología social analiza el proceso de envejecimiento y vejez desde una perspectiva interdisciplinaria que incluye dimensiones como la biológica psicológica social, educativa, política e histórica. El tema de los roles sociales es un tema de estudio situado específicamente en el campo de la Sociología del Envejecimiento y la Vejez, con algunos enfoques teóricos que se describen más adelante.
El tema de los roles sociales se ha destacado en instancias internacionales de reflexión y propuestas en torno al envejecimiento y la vejez. Tal es el caso de la Asamblea Mundial de Envejecimiento en Viena (United Nations 1982) y en Madrid 2002 (Naciones Unidas 2003), la Estrategia Regional de Implementación para América Latina y el Caribe del Plan de Acción Internacional de Madrid sobre Envejecimiento 2003, y, recientemente, la Convención Interamericana sobre la Protección de los Derechos Humanos de las Personas Mayores. Se enfatiza que desde la perspectiva de los derechos humanos se "reconoce las valiosas contribuciones actuales y potenciales de la persona mayor al bienestar común, a la identidad cultural, a la diversidad de sus comunidades, al desarrollo humano, social y económico y a la erradicación de la pobreza" (OEA 2015, 2). Todas estas instancias promueven la valoración y desarrollo de roles sociales activos para y con las personas mayores.

A su vez, desde una perspectiva teórica los roles sociales de las personas mayores se han estudiado principalmente en la Sociología del Envejecimiento y la Vejez ${ }^{1}$ (Moragas 2012; Piña y García 2016) por medio de enfoques teóricos situados, entre otras, en las perspectivas estructuralista funcionalista, interaccionismo simbólico y gerontología crítica (Piña y García 2016; Yuni 2015). Todas ellas subrayan el importante cambio que se produce en la vejez, en términos de lo que socialmente se espera que las personas mayores hagan en esta fase de la vida. Sin embargo, muchas de las teorías no consideran el hecho de que, más allá de su sustrato en un proceso biológico, el envejecimiento también está marcado por una lectura social que ocurre en un contexto de relaciones de asimetría y poder que determinan el lugar social asignado a la vejez y los espacios que las personas mayores pueden ocupar, así como los roles que les es posible adoptar. Asimismo, no se considera a la persona mayor como un actor central en la construcción de sus roles sociales, sino que se parte de la premisa de que los mismos deben ser definidos de forma externa -por ejemplo, mediante políticas sociales que incorporan de forma pasiva a las personas mayores sin considerar su contexto socio-cultural, instituciones que definen programas sociales que comprometen su autonomía, Establecimientos de Larga Estadía (ELEAM) que establecen su plan de trabajo sin consultarles previamente (Piña 2013) o programas recreativos sin una participación social activa que potencie sus vínculos con la comunidad.

Desde esa mirada, el presente trabajo pretende aportar a la discusión conceptual en torno al envejecimiento y género desde la perspectiva de los roles sociales, al proponer un enfoque de gerontología que enfatice la dimensión del envejecimiento y cultura como una construcción sociocultural, cuestionando los roles sociales y las desigualdades de género, y recuperando la idea de subjetividad y autonomía. El eje de la discusión es la temática de cuidados, argumentando que una (re)construcción sociocultural inclusiva de los roles sociales de las personas mayores debe pasar por la reflexión crítica en torno 
al cruce de roles de género y del imaginario cultural sobre el envejecimiento y sus potencialidades.

El trabajo está organizado de la siguiente manera: en el primer apartado, se sitúa teóricamente la discusión sobre los roles sociales en las personas mayores, argumentando que gran parte de la teoría social ha asumido que un problema a resolver en las sociedades modernas es la falta de roles en esta etapa de la vida. Contra este supuesto, se presentan algunas premisas de la gerontología rupturista que permiten repensar la (re)construcción de los roles sociales desde una óptica que no reproduzca desigualdades ya existentes en los ejes de género y edad. En el segundo apartado se introduce la cuestión de género, con base en la discusión la temática de los cuidados, en tanto un rol fuertemente diferenciado por género que suele atravesar toda la vida adulta de las mujeres y que resulta particularmente invisibilizado en la adultez mayor. Ello implica cuestionar la idea de la pérdida de roles y de los/as adultos mayores como únicamente receptores de cuidado. Pero, sobre todo, conlleva trasladar la discusión hacia quién -y cómo- se definen los roles socialmente aceptados para la vejez. Finalmente, se hace referencia a dos rupturas que, desde la gerontología rupturista, permitirían evitar los sesgos de género y edad implícitos en la visión dominante sobre la cuestión de los cuidados. Se plantea una propuesta como una posibilidad de cuestionar la mirada tradicional sobre los roles sin excluir a las personas mayores de este proceso de reconstrucción.

\section{Gerontología Social y Roles Sociales}

La gerontología social aborda temáticas centradas en envejecimiento y vejez, a través de su enfoque interdisciplinario (Piña, Olivo, Martínez y Mendoza 2018; Paola 2015; lacub y Arias 2017). Empero, para profundizar el tema de los roles sociales se recurre al campo del envejecimiento y la cultura por dos razones. Primero, los roles sociales se han problematizado desde el enfoque sociológico, presentando para este trabajo una breve descripción de los siguientes enfoques aplicados a la gerontología social: estructural -funcionalista; interaccionismo simbólico y crítico. Segundo, el presente artículo propone el enfoque de la gerontología rupturista, como una perspectiva crítica de abordaje de los roles sociales en un contexto sociocultural.

En relación con los enfoques sociológicos, en primer lugar, el enfoque abordaje funcionalista, en gerontología, señala que el envejecimiento implicaría una disminución de los roles sociales, surgiendo enfoques que destacan la inexistencia de roles sociales, rol sin rol y heterogeneidad de roles sociales (Piña 2015). En términos socioculturales, surgen actitudes e imágenes discriminatorias que asocian el significado de "viejo" a un estatus social negativo y poco valorado en la sociedad (Katz y Calasanti 2015; Clarke, Marshall, House y Lantz 2011; lacub y Arias 2017). Se destaca que las funciones sociales influyen en el autoconcepto y autoestima personal; es clave la socialización que facilita el proceso de adaptación interactiva de los individuos en su con- 
2. La ruptura epistemológica, planteada originalmente por Gastón Bachelard, ha sido vinculada a la gerontología por Piña (2015), que reconoce el aporte fundamental del pensador francés. texto. Los roles sociales son asignados por la sociedad y por los eventos propios asociados a la edad de los individuos (Piña y García 2016).

En segundo lugar, desde el interaccionismo simbólico, la teoría de la actividad menciona que las funciones sociales se pierden con la vejez, producto del alejamiento del trabajo. Así, mientras más activa permanezca la persona mayor, asumirá de mejor manera la vejez, sustituyendo sus roles sociales y favoreciendo un yo positivo (Piña 2015). La teoría de la continuidad destaca que no hay ruptura con los roles sociales de la fase adulta y lo que se produce son sólo algunos cambios asociados al proceso de adaptación que conlleva el envejecimiento y la vejez (Piña 2013). Ambos enfoques tienen en común que abordan el tema de los roles sociales: el primero lo considera como algo establecido socialmente, mientras el segundo los plantea como una construcción social a nivel individual, en un determinado contexto socio-cultural.

Este artículo opta por una tercera perspectiva: la de la gerontología crítica, que se centra en el contexto socio-cultural y su influencia en la construcción social de los roles sociales en las personas mayores como un proceso en permanente movimiento y elaboración. La gerontología crítica es una visión teórica que se basa en diversas tradiciones intelectuales de la teoría social crítica (Yuni 2015; Piña, Olivo, Martínez y Mendoza 2018). Dicha perspectiva conceptual no solo pretende comprender cómo se desarrolla la construcción social de la vejez, sino que, además, busca modificar tal visión mediante estrategias políticas y políticas públicas (Yuni, 2015; Piña, Olivo, Martínez y Mendoza, 2018).

Como parte de la gerontología crítica, el trabajo propone la mirada de la gerontología rupturista que conecta la perspectiva de la gerontología con la ruptura epistemológica ${ }^{2}$, entendida como un proceso que conlleva el tránsito de una visión conservadora de la vejez a una crítica, que propone una ruptura con el enfoque tradicional de la gerontología. Ello, de modo que permita repensar la (re)construcción de los roles sociales desde una óptica que no reproduzca desigualdades ya existentes en los ejes de género y edad.

Para el desarrollo de este análisis se propone considerar dos tipos de ruptura, que tienden a dominar la visión conservadora. En primer lugar, la experiencia básica, definida como un dominio de normas y prácticas naturalizadas, que no han sido sometidas a una reflexión crítica; en segundo lugar, el conocimiento general, entendido como teorías y enfoques conceptuales que se utilizan de forma acrítica y se repiten permanentemente en procesos de investigación e intervención con personas mayores. En el curso de este trabajo, se argumenta que la cuestión de las personas y los cuidados puede ser leída a partir de estas dos rupturas.

Desde tal enfoque, se cuestiona la inexistencia de roles sociales de las personas mayores y se propone una visión centrada en la "heterogeneidad de Roles Sociales en permanente construcción sociocultural" (Piña y García 2016), que planteando, asimismo, que la perspectiva de género es una temática fundamental de incluir en la discusión teórica del envejecimiento y los ro- 
les sociales. De hecho, la gerontología crítica asume que el proceso de envejecimiento está influido principalmente por el género, la clase socioeconómica, la etnia y las interacciones que se generan entre ellas, y se destaca que la pérdida de poder de las personas mayores se debe a las limitaciones estructurales en las que se desarrollan el envejecer (Herrera, Fernández y Barros 2018).

Existe aquí una afinidad conceptual con la interseccionalidad: la idea de que las identidades sociales y los roles asociados a ella -condicionados por género, etnia, clase social y edad, entre otras- no operan de manera independiente, sino que se articulan creando un sistema de opresión que refleja la intersección de múltiples formas de discriminación e invisibilización (Walby, Amstrong y Strid 2012). Este trabajo destaca los roles sociales de cuidados, ya que aparecen desigualdades tipificadas en el proceso de socialización de género y en constante regeneración en la vida cotidiana y curso de vida (Aguirre y Scavino 2016).

\section{Roles sociales, género y cuidado}

La cuestión de los roles sociales ha sido una preocupación permanente de la teoría de género. Específicamente, un tema central ha sido cómo la delimitación cultural de roles y funciones socialmente caracterizadas como "femeninas" y "masculinas" actúa como un principio de organización social, marcando las trayectorias vitales de hombres y mujeres de manera diferenciada (Wade y Ferree 2015). Esto es, la interpretación cultural de la diferencia biológica ha provisto repertorios de identidades (y roles asociados a ellos) distintos para las personas, según su sexo: típicamente, las labores de reproducción social y biológica -que incluye el trabajo doméstico y el cuidado de personas en situación de dependencia- han sido consideradas propias de las mujeres, puesto que se asume culturalmente que ellas estarían más capacitadas que los hombres para realizarlas como resultado de su capacidad biológica para procrear. Del mismo modo, se espera que los hombres asuman tareas propias del mundo del trabajo remunerado (Razavi y Staab 2014).

Dicha representación cultural ha sido criticada por la teoría de género por al menos tres razones: primero, por suponer una perspectiva binaria sobre las identidades (y las sexualidades), que niega las múltiples identidades que los seres humanos pueden construir. Segundo, por naturalizar las diferencias de roles sociales entre hombres y mujeres, en el sentido de suponer que son el resultado de la biología y de preferencias individuales, olvidando el papel crucial que procesos de socialización diferenciados por sexo tienen en la adopción de roles (Wade y Ferree 2015).

Y tercero, porque habitualmente la visión esencialista y binaria sobre la diferencia sexual introduce, además, una jerarquía, en la cual aquello que se considera masculino es más valorado socialmente que aquello que se considera femenino (Carrasco, Borderías y Torns 2011; Kenny 2007). Para la teo- 
ría de género, la cuestión del poder implícita en esta valoración diferenciada es muy importante porque revela como la asignación de roles opera no solamente como criterio de organización social, sino también de distribución de oportunidades de vida para ser y hacer lo que se escoja. Desde esta perspectiva, plantea no únicamente diferencia, sino desigualdad.

La cuestión de los cuidados es un ejemplo paradigmático de ese punto. Siguiendo a Aguirre, Batthyány, Genta y Perrota (2014), se entenderá el cuidado como la acción de ayudar a una persona en situación de dependencia en el desarrollo y el bienestar de su vida cotidiana. De esta manera incluye "hacerse cargo de los cuidados materiales, lo que implica un trabajo; de los cuidados económicos, lo que implica un costo económico, y de los cuidados psicológicos, lo que implica un vínculo afectivo, emotivo, sentimental" (Aguirre, Batthyány, Genta y Perrota 2014, 49).

Se trata de una actividad que puede ser remunerada (por ejemplo, el servicio doméstico), pero que generalmente se hace sin retribución económica cuando ocurre en el contexto de la familia. En América Latina, gran parte de este tipo de trabajo es realizado por mujeres, sin su respectivo pago (CEPAL 2016). Ello, como parte de una construcción social de la identidad femenina basada en la idea de la maternidad como aspiración central de las mujeres. Asociada a esta construcción identitaria está la representación del rol materno (cuidar, nutrir, proteger) como la principal contribución social de las mujeres (Gómez-Urrutia y Jiménez-Figueroa 2019; Razavi y Staab 2014).

Sin embargo, a pesar de tratarse de una labor indispensable para la continuidad de las sociedades y el bienestar de todos sus miembros, el trabajo de cuidados ha sido históricamente subsumido dentro de la esfera privada, sin que se lo considere un aporte social, sino más bien una actividad que las familias producen para sí mismas. Recientemente se le ha reconocido (al menos discursivamente) como trabajo socialmente valioso, tal como se considera al trabajo remunerado (Díaz-Fernández, Llorente-Marrón y Dema-Moreno 2018). No obstante, incluso cuando el trabajo doméstico y de cuidado se transa en el mercado de trabajo, tiene sueldos muy bajos, lo cual supone que -en la práctica- todavía se lo considera una actividad menos valiosa que otras (Carrasco, Borderías y Torns 2011).

Así, el rol de cuidadora asignado socialmente a las mujeres ha significado que, en la práctica, muchas veces se les imponga socialmente una tarea cuya contribución queda invisibilizada y subvalorada. Ello aumenta la probabilidad de que las mujeres que dedican su vida a cuidar de otros se encuentren en una situación de dependencia económica a lo largo de todo el curso de vida, ya que deben postergar otras actividades como el estudio y el trabajo remunerado en otras áreas para cumplir con las obligaciones familiares de cuidado. Lo anterior es crucial en contextos donde la mayoría de las recompensas materiales y la protección social se distribuyen a través de los vínculos con el mercado de trabajo, particularmente el formal (CEPAL 2016; Blofield y Martínez 2015). 
Reconociendo el importante aporte que la teoría de género ha hecho para iluminar el tema, esta perspectiva centró -desde sus orígenes- su preocupación en las mujeres en edad reproductiva, por el papel central que el control de la sexualidad y capacidad reproductiva femeninas (incluida la imposición del ideal materno) ha tenido en la limitación de las oportunidades de vida de las mujeres (Silver 2003). Cuando se plantea la necesidad de reconocer el cuidado como una contribución social, el foco tiende a ponerse en los niños, niñas y adolescentes, o en las propias personas mayores en cuanto dependientes. Esto es, se asume que las mujeres mayores -por haber dejado atrás la edad reproductiva- ya no tienen responsabilidades familiares de cuidado. Con ello, implícitamente se presupone que se han quedado "sin rol" por ser personas mayores.

No obstante, los datos muestran una realidad distinta. Por ejemplo, la IV Encuesta de Calidad de Vida en la Vejez (Universidad Católica- Caja los Andes 2017) muestra que, en Chile, entre quienes tienen nietos/as, 15,4\% de las personas mayores cuidó de ellos/as todos los días de la semana, y $8,5 \%$ lo hizo varias veces por semana. Las mujeres entre 60 y 75 años son quienes más están al cuidado de los nietos diariamente. De tal manera, las mujeres mayores proveen un apoyo crucial para que las generaciones más jóvenes (especialmente sus hijas y nueras) puedan compatibilizar obligaciones laborales y familiares cuando existen pocos mecanismos colectivos para ese propósito, como salas-cuna públicas o servicios privados de cuidados de calidad que las personas puedan costear.

De manera similar, de acuerdo con la Encuesta de Caracterización Socioecónomica Nacional de Chile, CASEN, de 2015, un 30,7\% de los/as cuidadores/as de personas de 60 años y más con dependencia funcional que recibe asistencia personal por alguien del hogar tiene entre 60 y 74 años esto es, se trata de adultos mayores cuidando de otras personas mayores, muy frecuentemente sus parejas. El único otro grupo etario de cuidadores/as más numeroso que el mostrado anteriormente, es el que va de los 45 a los 59 años -hijas y nueras, principalmente, con un 34,4\% (Ministerio de Desarrollo Social de Chile 2017).

Así, una parte importante del cuidado de adultos mayores es provista por otras personas dentro del mismo grupo etario. Datos similares son aportados en otros países latinoamericanos con índices de envejecimiento importantes, como Costa Rica (Fernández-Rojas y Robles 2007, 37 y ss.) y Uruguay (Batthyány 2008), así como en países ya avanzados en la transición demográfica fuera de la región, como es el caso de España (Instituto de Mayores y Servicios Sociales de España 2017) y el Reino Unido (Greenwood y Smith 2016), que corroboran el aporte realizado por cuidadoras de 60 años y más, a menudo sin reconocimiento ni apoyo de la política pública.

Tal invisibilización del cuidado a lo largo de la vida presenta una paradoja: de un lado, refuerza tácitamente los roles tradicionales, pues asume que las mujeres harán la labor de cuidado sin recompensa o reconocimiento a lo largo de toda la vida porque la sociedad espera que lo hagan. Del otro lado, 
se ignora las contribuciones de los y las adultas mayores en otras áreas importantes para el bienestar social, como el cuidado y la participación social y comunitaria, en el caso del cual se ocupa el presente trabajo. $Y$ lo hace doblemente en el caso de las mujeres, en la intersección entre género y vejez. Se presenta dicha fase de la vida como una en la que no hay roles definidos $y$, por ello, disminuye la contribución social que los individuos pueden hacer, aunque en la práctica no sea así.

lluminar este hecho se relaciona con la necesidad de contar con una mirada crítica respecto de cómo se construyen los roles de género no solo en la edad reproductiva, sino también cuando esa etapa ha terminado -lo cual es biológicamente más evidente para las mujeres, por el hito representado por la menopausia. El rol de cuidadora, para las mujeres, traspasa con mucho la edad reproductiva; pero a medida que avanza el ciclo vital queda invisibilizado no por el género únicamente, sino por una construcción de la vejez que supone que las personas pierden sus roles al llegar a la vejez, por lo cual deben "llenar" el espacio dejado por el rol que tenían antes de alcanzar la adultez mayor como la única forma de mantenerse socialmente integradas -en el mejor de los casos- o para no representar una "carga" para las personas más jóvenes, en el otro extremo.

Tal idea convive con un discurso sobre la vejez activa que, al menos en el contexto latinoamericano, tiende a identificar la nueva forma de percibir la vejez con la generación de ingresos en el mercado laboral o con formas de participación que contribuyan a no agravar los sistemas de seguridad social y salud, fenómeno ya identificado en otros contextos (Biggs, Carr y Haapala 2015).

Lo anterior, lógicamente, no significa argumentar que los roles sociales no cambian a medida que las personas transitan por diversas etapas de la vida. Significa, más bien, subrayar que resulta problemático asumir que una vez que los roles propios de la adultez -como el trabajo remunerado o el ejercicio de la maternidad/paternidad- pierden importancia relativa, se produce un "vacío" o que resulta imposible "renegociar" roles desde las aspiraciones de las personas mayores. Ello porque, como apuntan Aguirre y Scavino (2016) y Silver (2003), la vejez puede ser una etapa de reinvención social, tanto para hombres como para mujeres, precisamente porque las presiones sociales para la definición de una identidad de género tradicional son menores (aunque no desaparezcan).

Lo anterior podría significar la oportunidad de retomar proyectos postergados por las responsabilidades familiares y laborales, generar otros nuevos que resultaban difíciles de realizar en una etapa anterior de la vida o resignificar roles tradicionales, desde la perspectiva de que sean una opción, y no una imposición social.

Visibilizar dicha posibilidad, sin embargo, significa cuestionar supuestos epistemológicos importantes sobre la vejez y el rol de las personas mayores en nuestra sociedad, que hoy pueden ser un obstáculo para la realización efectiva de los derechos que, gradualmente, han ganado (al menos formalmente) 
en el plano internacional, como el derecho a ser cuidados. Pero también significa reconocer sus aportes sociales, ya sea en lo familiar o en lo comunitario, donde la manutención de redes de apoyo recíprocas es de particular importancia. Esta cuestión será abordada en la sección siguiente.

\section{Repensar la vejez: la propuesta de la gerontología rupturista}

Como ya se ha señalado, abordar la cuestión de los roles sociales en la vejez de manera crítica requiere examinar supuestos tanto referidos al género como a la contribución social de las personas mayores. Desde la lectura propuesta, a continuación, se hará referencia a dos rupturas que, desde la Gerontología Rupturista, permitirían evitar los sesgos de género y edad implícitos en la visión dominante sobre la cuestión de los cuidados. A partir de ahí, es posible plantear una propuesta que se presenta como una oportunidad de cuestionar la mirada tradicional sobre los roles sin excluir a las personas mayores de este proceso de reconstrucción. Ello en el entendido de que más que una búsqueda social de roles, las personas mayores necesitan contar con opciones de vida entre las cuales puedan escoger.

La primera de esas rupturas es con la experiencia básica, cuando se antepone y está por encima de la crítica en el proceso de investigación e intervención gerontológica, que se centra en una visión tradicional de los roles sociales y que, en el caso de los cuidados, socialmente tiende a naturalizar dicha tarea como un rol de mujeres (Carrasco, Borderías y Torns 2011; Aguirre y Scavino 2016). Además, como una parte significativa de este trabajo no se transa en el mercado laboral, tiende a vérselo como no-trabajo, como inactividad (Aguirre y Scavino 2016) o como una función improductiva (Piña y García 2016).

Para superar dicha situación, se requiere gatillar una ruptura en las políticas públicas (por ejemplo), de manera que las mismas reconozcan el valor social de los aportes no monetarizados que se hacen por medio del trabajo de cuidados, a nivel familiar y comunitario, como una función productiva y necesaria para el bienestar social. En otras palabras, es deseable que hombres y mujeres mayores tengan la oportunidad de escoger este tipo de actividad porque de ese modo contribuyen a sus comunidades con su capacidad y experiencia, y no solo porque los mantiene socialmente integrados(as).

Se trata de una relación de beneficio recíproco, en la que resulta crucial el incentivo a que los hombres asuman más labores de cuidados, en la lógica de redistribuir esa responsabilidad más equitativamente en todas las etapas de la vida. Así pues, la gerontología rupturista modificaría la visión tradicional de los roles sociales instalado en los planes, programas y proyectos con personas mayores, profundizando e incorporando la dimensión de género en el análisis gerontológico actual.

La segunda ruptura es el conocimiento general, que surge cuando se considera un enfoque teórico como inmune a los cuestionamientos y se aplica de forma permanente, sin considerar que se debe ajustar al contexto sociocultu- 
ral de cada situación. Por ello, es fundamental que los conceptos se flexibilicen (Piña 2015). En este sentido, se refuerza la necesidad de modificar el enfoque actualmente vigente de la vejez como un "problema", definido desde una mirada externa, complementándolo con una visión gerontológica que lo plantee también como posibilidad. Ello permitiría analizar los roles sociales bajo una perspectiva de gerontología rupturista, rechazando la idea de la inexistencia de roles sociales en las personas mayores y destacando que son heterogéneos y en permanente construcción sociocultural. Con ello, se subraya la importancia de permitir que las personas mayores participen en la redefinición de sus propios roles y contribuciones a la sociedad, siendo actores sociales protagónicos y no solamente beneficiarios de las acciones gerontológicas que se desarrollen.

Lo anterior, no obstante, también significa reconocer que -de la misma forma que ocurre con el género- las definiciones sociales que se hacen del proceso biológico de envejecer se dan en el contexto de relaciones de poder. Desde esa mirada, en las sociedades en las cuales envejecer sea percibido de manera negativa, la vejez puede significar, además de la invisibilización del rol, la pérdida de prestigio y de poder. Si a las personas mayores se les limitan los espacios sociales en los cuales contribuyen -esto es, si se les discrimina o se les señala como una "carga" presente o futura- posiblemente verán disminuida su capacidad de agencia y, con ello, los márgenes disponibles para decidir su propio destino.

Esta idea, como observan Andrews (2009) y Katz y Calasanti (2015), es frecuentemente obviada en los discursos del envejecer con éxito (succesful aging) porque obscurece el hecho de que el proceso de envejecimiento, desde su vertiente social, está marcado por las oportunidades de vida y las desigualdades que las personas han debido enfrentar durante sus trayectorias vitales.

Bajo ese prisma, las oportunidades que los sujetos tengan de decidir respecto de su propia biografía pueden ser determinantes para definir si el cuidado de otros/as se vive como una actividad gratificante, llena de sentido y que permite mantenerse integrado(a), o -por el contrario- como una imposición externa de roles, proceso que típicamente asigna dicha tarea a quienes tienen menos poder, como grupo social (Silver 2003). Lo antes dicho, podría ser el caso de políticas que asumen que las mujeres mayores deben mantener su rol de cuidadoras habituales como fórmula para facilitar que las mujeres más jóvenes se incorporen al mercado de trabajo remunerado, en lugar de enfrentar la cuestión promoviendo la participación más activa de los padres (hombres) y del Estado en el cuidado infantil.

En tal caso, el cuidado podría volver a ser una imposición social, en lugar de ser una opción que se signifique positivamente en el contexto de la propia biografía -por ejemplo, elegir minimizar su rol de cuidadoras para asumir otros papeles, cuidar con más experiencia o sin las presiones de la crianza de los(as) propios(as) hijos(as)- o escogida, en un contexto donde haya otras posibilidades de vincularse socialmente (estudiar, trabajar remuneradamente o dedicar más tiempo al ocio). 
Así, la vejez también puede ser el escenario de reconstrucción de roles de género que pueden alterar, pero también reproducir, relaciones de poder entre los sexos y entre las generaciones. Organizar socialmente el cuidado de una manera más equitativa implica negociaciones con los ideales de femineidad y masculinidad dominantes, aunque, adicionalmente, supone balancear las necesidades de los distintos grupos etarios.

De ahí la importancia de combinar la producción de datos cuantitativos sobre la calidad de vida de las personas mayores, sus actividades principales y sus necesidades de cuidado con el estudio de percepciones y narrativas personales sobre estas mismas materias. El cuidado de otros/as puede ser incorporado en las narrativas personales de hombres y mujeres de formas variadas, dependiendo de la capacidad que los sujetos tengan para negociar sus márgenes de autonomía personal en función de criterios de estratificación social como género, etnia o clase social.

\section{Conclusiones}

Producto de la reflexión sobre los roles sociales expuesta en las páginas precedentes, surge la necesidad de levantar información -a partir de las propias personas mayores- sobre la valoración subjetiva que los individuos realizan de la situación social en que los pone la vejez, en términos del lugar social que pasan a ocupar al entrar en la categoría de personas mayores (incluyendo diferencias de género) y las potencialidades de esta fase de la vida. Como se ha argumentado a lo largo del trabajo, lo anterior implica recuperar la preocupación teórica por la forma en que se construyen los roles sociales para diferentes grupos. En este punto la teoría de género ha hecho un aporte fundamental al problematizar cómo la diferencia anatómica entre hombres y mujeres ha sido culturalmente interpretada en un contexto de relaciones de poder, en el cual dicho poder se expresa -entre otras formas- en la capacidad de definir el lugar que los sujetos ocupan en la estructura social y sus roles. Dicha intuición básica se intersecta con el interés de la gerontología social por la forma en que el envejecimiento de los cuerpos se significa socialmente. Aquí las posibles asimetrías de poder -en este caso, entre las generaciones- asimismo, deben ser consideradas, si se desea que la vejez sea una etapa de la vida en la cual los sujetos puedan seguir descubriendo y desarrollando sus capacidades.

Lo anterior resulta particularmente relevante en países como los latinoamericanos, caracterizados por redes de protección social más bien frágiles, y donde la contribución de las redes familiares y comunitarias es clave para el bienestar de todos los grupos sociales (Blofield y Martínez 2014). Las cifras disponibles sugieren que las personas mayores son muy activas en estos espacios, constituyendo un potencial que debe valorarse y que podría extenderse a otros ámbitos. Sin embargo, para que ocurra es también necesario promover una mayor igualdad de oportunidades entre hombres y mujeres a lo largo de la vida. 
A partir de esa perspectiva, es deseable que investigaciones futuras sobre envejecimiento y roles sociales levanten información sobre la visión subjetiva de las personas mayores, cuyos ámbitos de análisis consideren su rol como receptores de cuidado, pero también como proveedores del mismo para otras personas mayores o en situación de dependencia, o bien en la recuperación de roles comunitarios.

En ese marco, la reflexión busca aportar al andamiaje conceptual sobre el acelerado proceso de envejecimiento que viven muchas sociedades y que vuelve a plantear el desafío de asegurar derechos de una manera que no acabe imponiendo a las personas definiciones cruciales para sus vidas.

La propuesta pretende contribuir a los planteamientos de la Convención interamericana sobre la protección de los derechos humanos de las personas mayores, que indica que "los Estados Parte deberán adoptar medidas tendientes a desarrollar un sistema integral de cuidados que tenga especialmente en cuenta la perspectiva de género y el respeto a la dignidad e integridad física y mental de la persona mayor" (OEA 2015, 11).

Asimismo, en instancias internacionales de reflexión y propuestas en torno al envejecimiento se ha destacado la necesidad de potenciar a las personas mayores en todos los ámbitos de la vida social, incluyendo la categoría de género. Tal es el caso de las Asambleas Mundiales, la Estrategia Regional de Implementación para América Latina y el Caribe del Plan de Acción Internacional de Madrid sobre el Envejecimiento y la Declaración de Brasilia.

En esta última, se menciona que "Respaldamos activamente la incorporación de la perspectiva de género en todas las políticas y programas que tengan en consideración las necesidades y experiencias de las personas de edad" (Cepal 2007, 3). En esa línea, una idea central para los propósitos enunciados en este trabajo es la relevancia de incorporar la perspectiva de género en todas las políticas y programas dirigidos a hacer efectivos los derechos de la persona mayor, al tiempo que se destaca la necesidad de reconocer los mecanismos de poder que operan en la intersección entre género y edad. Lo anterior requiere una mirada crítica de la opinión especializada y pública sobre los roles sociales de las personas mayores, para evitar los sesgos que se han discutido a lo largo de estas páginas.

Como limitación del trabajo, se plantea que ello requerirá una reflexión más amplia respecto de cómo traducir la mirada mostrada a categorías empíricas que permitan conocer la subjetividad de las personas mayores y sus aspiraciones en el plano de los roles, y cómo estas aspiraciones están cruzadas por clivajes sociales como género y clase social. Lo anterior, en la lógica de que más allá del inevitable y común proceso biológico que lleva al envejecimiento, las posibilidades de envejecer de una forma subjetivamente satisfactoria se distribuyen también socialmente. 


\section{Referencias}

Andrews, Molly. 2009. "The Narrative Complexity of Successful Ageing». International Journal of Sociology and Social Policy 29(1/2): 73-83. Disponile en https://www.emeraldinsight.com/doi/10.1108/01443330910934736

Aguirre, Rosario y Sol Scavino. 2016. «Cuidar en la Vejez: Desigualdades de Género en Uruguay». Papeles del CEIC 1 (150): 1-41. Disponible en http://dx.doi.org/10.1387/pceic.15449.

Aguirre, Rosario, Karina Batthyány, Natalia Genta y Valentina Perrota. 2014. «Los cuidados en la agenda de investigación y en las políticas públicas en Uruguay». Revista de Ciencias Sociales Íconos 50: 43-60.

Batthyány, Karina. 2008. Género, cuidados familiares y uso del tiempo. Informe final de investigación. Montevideo, UNIFEM. Acceso el 15 de marzo de 2019. www.cienciassociales.edu.uy/wpcontent/uploads/sites/3/2013/archivos/Karina\%20Batthy\%C3\%A1ny \%20G\%C3\%A9nero,\%20cuidados $\% 20$ familiares $\% 20 y \% 20$ uso $\% 20$ del \%20tiempo.pdf.

Biggs, Simon, Ashley Carr e Irja Haapala. 2015. "Work, aging, and risks to family life: The case of Australia". Canadian Journal on Aging-Revue Canadienne $d u \quad$ Vieillissement $34 \quad$ (3): 321-330. doi:10.1017/S0714980815000185.

Blofield, Merike y Juliana Martínez Franzoni. 2015. «Maternalism, CoResponsibility, and Social Equity: A Typology of Work-Family Policies». Social Politics 22(1): 38-59.

Carrasco, Cristina, Cristina Borderías y Teresa Torns. 2011. «Introducción». En El trabajo de cuidados: Antecedentes históricos y debates actuales, editado por Cristina Carrasco, Cristina Borderías y Teresa Torns, 13-95. Madrid: Los libros de la Catarata.

Comisión Económica para América Latina y el Caribe. 2007. Declaración de Brasilia. Segunda Conferencia Regional Intergubernamental sobre Envejecimiento en América Latina y el Caribe: hacia una sociedad para todas las edades y de protección social basada en derechos. Acceso el 26 abril de 2019. https://www.cepal.org/es/publicaciones/21505declaracion-brasilia-segunda-conferencia-regional-intergubernamental

Comisión Económica para América Latina y el Caribe. 2016. Autonomía de las mujeres e igualdad en la agenda de desarrollo sostenible. Santiago: Organización de las Naciones Unidas.

Clarke, Philippa, Víctor Marshall, James House y Paula Lantz. 2011. «The social structuring of mental health over the adult life course: advancing theory in the sociology of aging». Social Forces 4: 1287-1313.

Díaz-Fernández, Montserrat, Mar Llorente-Marrón y Sandra Dema-Moreno. 2018. «Tratamiento económico y modelización del cuidado». 
Convergencia, Revista de Ciencias Sociales 76: 37-55. Disponible en https://doi.org/10.29101/crcs.v25i76.4347

Herrera, María Soledad, María Beatriz Fernández y Carmen Barros. 2018. «Estrategias de afrontamiento en relación con los eventos estresantes que ocurren al envejecer». Ansiedad y estrés 24, 47-52. Disponible en https://doi.org/10.1016/j.anyes.2017.10.008

lacub, Ricardo y Claudia Arias. 2017. «La gerontología comunitaria: poder, comunidad y vejez». En Gerontología. Actualizaciones y temas emergentes. Editado por Ingrid Fergusson y Claudio Rojas, 45-65. Talca: Universidad Católica del Maule.

Instituto de Mayores y Servicios Sociales de España. 2017. Informe 2016: Las personas mayores en España. Acceso el 23 de abril de 2019. http://www.imserso.es/imserso_01/documentacion/estadisticas/informe ppmm/index.htm

Fernández-Rojas, Xinia y Arodys Robles. (2007). Primer Informe de Situación de la Persona Adulta Mayor en Costa Rica. Disponible en doi: 10.13140/RG.2.1.3344.7123

Gómez-Urrutia, Verónica y Andrés Jiménez-Figueroa, A. 2019. «Género y trabajo: hacia una agenda nacional de equilibrio trabajo-familia en Chile». Revista de Ciencias Sociales Convergencia: 79: 1-24. https://doi.org/10.29101/crcs.v0i79.10911

Greenwood, Nan y Raymond Smith. 2016. "The oldest carers: A narrative review and synthesis of the experiences of carers aged over 75 years» Maturitas 94: 161-172. Disponible en http://dx.doi.org/10.1016/j.maturitas.2016.10.001

Katz, Stephen y Toni Calasanti. 2015. «Critical perspectives on successful aging: does it "appeal more than illuminates?». The Gerontologist 55(1): 26-33. Disponible en https://doi.org/10.1093/geront/gnu027.

Kenny, Meryl. 2007. «Gender, Institutions and Power: A Critical Review». Politics 27(2): 91-100. Disponible en https://doi.org/10.1111/j.14679256.2007.00284.x

Ministerio de Desarrollo Social. 2017. Síntesis de resultados Encuesta de Caracterización Socioeconómica CASEN - Adultos Mayores 2015. Acceso el 10 de agosto 2018. Disponible en http://observatorio.ministeriodesarrollosocial.gob.cl/casenmultidimensional/casen/docs/CASEN_2015_Resultados_adultos_mayo res.pdf.

Moragas Moragas, Ricardo. 2012. Jubilación siglo XXI. Madrid: Editorial Palibrio Spain. 2012.

Naciones Unidas. 2003. Declaración Política y Plan de Acción Internacional de Madrid sobre el Envejecimiento. Acceso el 8 de septiembre de 2017. 
Disponible en https://social.un.org/ageing-workinggroup/documents/mipaa-sp.pdf.

Organización de los Estados Americanos. 2015. Convención interamericana sobre la protección de los derechos humanos de las personas mayores. Acceso el 10 de agosto de 2018. Disponible en http://www.oas.org/es/sla/ddi/tratados multilaterales interamericanos_a -70 derechos humanos personas mayores.asp.

Paola, Jorge. 2015. «Hacia una intervención crítica del Trabajo Social en el campo gerontológico"». En Más mayores, más derechos. Diálogos interdisciplinarios sobre vejez, compilado por Jorge Paola, María Tordo y Paula Danel, 129-160. La Plata: Editorial de la Universidad Nacional de la Plata.

Piña Morán, Marcelo. 2013. Gerontología social aplicada: Visiones estratégicas para el trabajo social. Buenos Aires: Editorial Espacio. 2013.

Piña Morán, Marcelo. 2015. «La Formación del Espíritu Científico en el Trabajo Social y la Vigilancia Epistemológica en el Campo Gerontológico». En Más mayores, más derechos. Diálogos interdisciplinarios sobre vejez, compilado por Jorge Paola, María Tordo y Paula Danel, 227-258. La Plata: Editorial de la Universidad Nacional de la Plata.

Piña Morán, Marcelo y Leidy García Perez. 2016. "«Socio-cultural connections and ruptures: social roles of older adults in Chile"». Journal of Population Ageing 9. 263-280. Disponible en https://doi: 2016; 10.1007/s12062-016-9142-6

Piña, Marcelo, María Gladys Olivo, María de la Luz Martínez y Víctor Mendoza. 2018. «Intervención situacional gerontológica: estrategias ara potenciar los roles sociales de los mayores». Revista Médica del Instituto Mexicano del Seguro Social 56 (S1): 102-109.

Razavi, Shahra y Silke Staab. 2014. Global Variations in the Political and Social Economy of Care. Londres: Routledge/UNRISD Research in Gender and Development.

Silver, Catherine. 2003. "Gendered identities in old age: Toward (de)gendering?». Journal of Aging Studies 17 (4): 379-397. Disponible en https://doi.org/10.1016/S0890-4065(03)00059-8.

United Nations. 1982. International Plan of Action on Ageing. Acceso el 8 de septiembre de $2017 . \quad$ Disponible en http://www.msal.gov.ar/ent/images/stories/programas/pdf/201308 planaccion- envejecimiento-viena-1982.pdf.

Universidad Católica de Chile- Caja los Andes. 2017. IV Encuesta de Calidad de Vida en la Vejez. Acceso el 8 de noviembre de 2018. Disponible en http://www.senama.gob.cl/storage/docs/Chile-y-sus-Mayores-10-anosde-Encuesta-Calidad-de-Vida-en-la-Vejez-2016.pdf. 
Wade, Lisa y Myra Marx Ferree. 2015. Gender: Ideas, interactions, institutions. Nueva York: W.W. Norton \& Co.

Walby, Sylvia, Jo Armstrong y Sofia Strid. 2012. «Intersectionality: Multiple Inequalities in Social Theory». Sociology 46 (2): 224-240. Disponible en https://doi.org/10.1177/0038038511416164.

Yuni, José. 2015. «Perspectivas críticas acerca de la construcción social de la vejez y las intervenciones socio-culturales"» En Más mayores, más derechos. Diálogos interdisciplinarios sobre vejez, compilado por Jorge Paola, María Tordo y Paula Danel, 320-346. La Plata: Editorial de la Universidad Nacional de la Plata. 